Review Article

\title{
Diagnosing Paroxysmal Atrial Fibrillation: Are Biomarkers the Solution to This Elusive Arrhythmia?
}

\author{
P. J. Howlett, ${ }^{1}$ F. S. Hatch, ${ }^{1}$ V. Alexeenko, ${ }^{1}$ R. I. Jabr, ${ }^{1}$ E. W. Leatham, ${ }^{2}$ and C. H. Fry ${ }^{3}$ \\ ${ }^{1}$ School of Biosciences and Medicine, The University of Surrey, Guildford GU2 7XH, UK \\ ${ }^{2}$ Department of Cardiology, The Royal Surrey County Hospital, Guildford GU2 7XX, UK \\ ${ }^{3}$ School of Physiology and Pharmacology, The University of Bristol, Bristol BS8 1TD, UK \\ Correspondence should be addressed to P. J. Howlett; philippah@doctors.org.uk
}

Received 26 December 2014; Revised 9 February 2015; Accepted 12 February 2015

Academic Editor: Michele Miragoli

Copyright (C) 2015 P. J. Howlett et al. This is an open access article distributed under the Creative Commons Attribution License, which permits unrestricted use, distribution, and reproduction in any medium, provided the original work is properly cited.

\begin{abstract}
Atrial fibrillation (AF) is the commonest sustained arrhythmia globally and results in significantly increased morbidity and mortality including a fivefold risk of stroke. Paroxysmal atrial fibrillation (PAF) constitutes approximately half of all AF cases and is thought to represent an early stage of the disease. This intermittent form of atrial arrhythmia can be a challenge to identify and as a result many affected individuals are not prescribed appropriate antithrombotic therapy and hence are at risk of stroke and thromboembolism. Despite these adverse outcomes there have been relatively few diagnostic advances in the field since the introduction of the Holter monitor in 1949. This review aims to establish the available evidence for electrophysiological, molecular, and morphological biomarkers to improve the detection of PAF with reference to the underlying mechanisms for the condition.
\end{abstract}

\section{Introduction}

Atrial fibrillation (AF) is the most frequently encountered cardiac arrhythmia globally, affecting $2 \%$ of the general population and rising to $10 \%$ of those aged over 80 years. One in four individuals will experience AF in their lifetime [1]. By 2050 the prevalence of AF is expected to increase by threefold and this poses a considerable public health concern [2]. Overall AF exacerbates the risk of stroke and is associated with a twofold excess mortality with $20 \%$ of all strokes being as a result of $\mathrm{AF}$ and 1 in 5 patients first presenting with $\mathrm{AF}$ in the context of cerebral ischaemia [3]. In addition AF is also linked to larger strokes based on both clinical and radiological findings [4]. Despite these risks, it has been estimated that at least $20 \%$ of cases of AF remain undiagnosed and are not prescribed appropriate stroke prevention therapy [5].

Paroxysmal atrial fibrillation (PAF) is thought to constitute between 25 and $60 \%$ of cases of AF [6] and is thought to precede sustained $\mathrm{AF}$, culminating in progressive atrial electrical and structural remodelling, otherwise coined "AF begets AF." PAF has been defined by the American Heart
Association as "recurrent (two or more) episodes of AF that terminate spontaneously lasting between 30 seconds and less than seven days" [7]. An example of the initiation of PAF is seen in Figure 1.

The true prevalence of PAF is unknown due to asymptomatic episodes and a low yield afforded by conventional monitoring. However between 6 and 28\% of cryptogenic strokes have been found to be secondary to PAF [8] and PAF conveys an equivalent risk of stroke to sustained forms of AF [9]. Unfortunately PAF, relative to the other forms of AF termed "persistent" and "permanent," can be a particular challenge to diagnose due to the variable onset of the arrhythmia, potential brevity, and also frequent lack of symptoms [10].

\section{Pathophysiology of Atrial Fibrillation}

The cardiac action potential (AP) is a key determinant of cardiac electrical activity and results from transmembrane ion fluxes through ion channels and transporters. A schematic representation of a human atrial action potential 


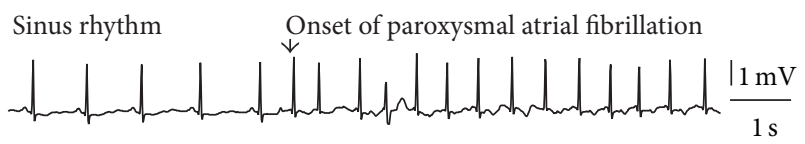

FIGURE 1: An ECG recording from a continuous cardiac monitor from a patient suffering from paroxysmal atrial fibrillation (PAF) lasting for several hours. The arrow marks the onset of AF, characterised by a variable R-R interval (representing the time between two successive ventricular contractions) and loss of P-waves (absence of coordinated atrial activity) (unpublished).

and the principal currents involved is shown in Figure 2(a). The resting potential is maintained at about $-80 \mathrm{mV}$ via $\mathrm{K}^{+}$ equilibrium potential. Upon activation, rapid depolarization occurs by a large inward $\mathrm{Na}^{+}$current $\left(I_{\mathrm{Na}}\right)$ and an inward $\mathrm{Ca}^{2+}$ current via L-type $\mathrm{Ca}^{2+}$ channels $\left(I_{\mathrm{CaL}}\right)$ resulting in the plateau phase. As the $\mathrm{Ca}^{2+}$ current declines the AP repolarizes to the resting potential.

Arrhythmia generation in the left atrium (LA) originates from a combination of abnormalities in impulse initiation, impulse conduction, or a combination of the two [11]. Abnormal impulse initiation can be subclassified further due to enhanced automaticity or triggered activity. Enhanced automaticity (ectopic arrhythmias) may be produced by irregular intracellular $\mathrm{Ca}^{2+}$ release. Similarly triggered activity, caused by a spontaneous inward current, generates secondary afterdepolarizations early in the plateau phase (EADs) or upon repolarisation (DADs) (Figure 2(b)). Alternatively due to abnormal AP conduction, reentrant arrhythmias occur, preventing the normal pattern of propagation across the myocardium. This is attributable to a combination of alteration of the electrical properties of intercellular gap junctions; structural remodelling of the myocardium; and generation of EADs/DADs that per se have slower conduction. Both mechanisms may be present simultaneously where ectopic activity begets reentrant arrhythmias resulting in self-perpetuation.

The former described electrophysiological mechanisms are coupled to atrial electrical remodeling, characterised by changes in atrial refractoriness and slowed conduction time. These changes occur due to alterations in AP currents, especially $\mathrm{Ca}^{2+}$ influx and its subsequent homeostasis, and provide ongoing atrial arrhythmogenic substrates [12].

Atrial structural remodeling is typified by cardiac fibrosis, characterized by accumulation of collagenous material in the extracellular space (Figure 3), and is proposed to widen gap junctions and decrease communication between cardiomyocytes. Reduced AP conduction velocity is directly correlated to the extent of fibrosis and is linked to persistent reentrant circuit arrhythmias [13]. Angiotensin II produced by fibroblasts exacerbates the situation to increase cellular proliferation and cardiac fibrosis [14]. Alternatively structural remodeling may arise due to the release of proinflammatory cytokines after surgery or injury and the interaction of inflammatory proteins with angiotensin II promotes further atrial fibrosis [15]. This inflammatory response has been implicated in the aetiology of AF although the question remains whether this is attributable to the arrhythmia itself or another underlying disease state [16].
The ultimate consequences of $\mathrm{AF}$, stroke and systemic embolism, result from the dislodgement of LA thrombi. Thrombus is most commonly located in the left atrial appendage (LAA) [17], a small pouch found in the LA (Figure 4). The generation of thrombi is triggered by atrial stasis, as a result of impaired atrial contraction due to atrial arrhythmia. Other factors promoting thrombus formation include damage to the atrial wall and a hypercoagulable state [18].

\section{Current Diagnostics for Paroxysmal Atrial Fibrillation}

Current methods to diagnose PAF are limited to electrocardiogram (ECG) analysis, which may fail to document an episode of AF if occurring outside the monitoring period. Recent trials have highlighted that prolonged cardiac monitoring detects significantly more cases of PAF in the cryptogenic stroke population than using a conventional 24hour Holter monitor $[19,20]$. Clinical risk factors for AF are widely recognized and models have been proposed to predict incident AF using a number of clinical variables. Such a risk score devised by the Framingham Heart Study is based on a number of simple clinical parameters including age, gender, a significant murmur, cardiac failure, systolic blood pressure, hypertension, body mass index, and PR interval. The score yielded a C-statistic of 0.78 (95\% CI 0.76-0.80), with a Cstatistic being a measure of a model's predictive power and a value of over 0.7 considered to be reasonable. The addition of echocardiographic measurements further improved this to 0.79 [21]. There is however a lack of diagnostic armoury to detect PAF. This is in stark contrast to other cardiovascular conditions, for example, coronary artery disease and cardiac failure, with a comparable disease prevalence and routine use of diagnostic biomarkers [22].

\section{Biomarkers}

A biomarker can be defined as "a characteristic that is objectively measured and evaluated as an indicator of normal biological processes, pathogenic processes, or pharmacologic responses to a therapeutic intervention" [23]. Biomarkers not only have the potential to identify a disease process but also provide valuable information about underlying disease mechanisms and, as a consequence, potential therapeutic targets.

Biomarkers have been proposed as a tool to predict onset of AF in a variety of settings including at initial presentation, following cardiothoracic surgery, recurrence after cardioversion and ablation, and in the event of a cryptogenic stroke. Additionally, accumulating research has shown that biomarkers could be used to predict the transition of PAF to sustained AF, as well as, alongside conventional risk scores, to determine thromboembolic risk $[24,25]$. The LA has been the main focus for identifying biomarkers for PAF given the inextricable association between this chamber and the disease process itself. As such potential biomarkers to detect PAF can be broadly categorised into electrophysiological, molecular, and morphological indices. 


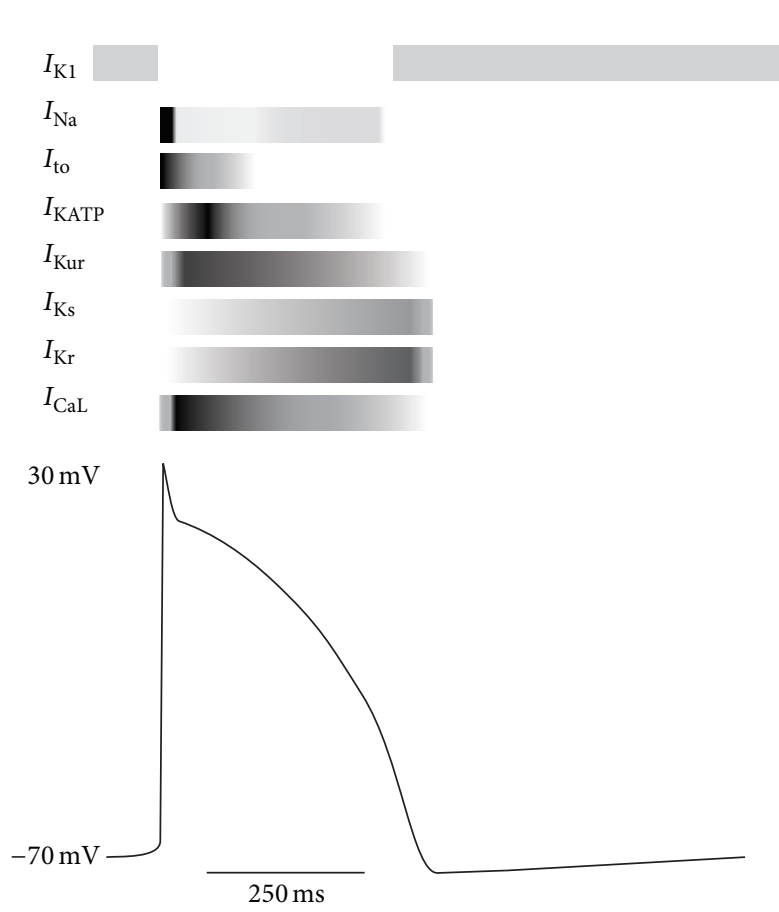

(a)

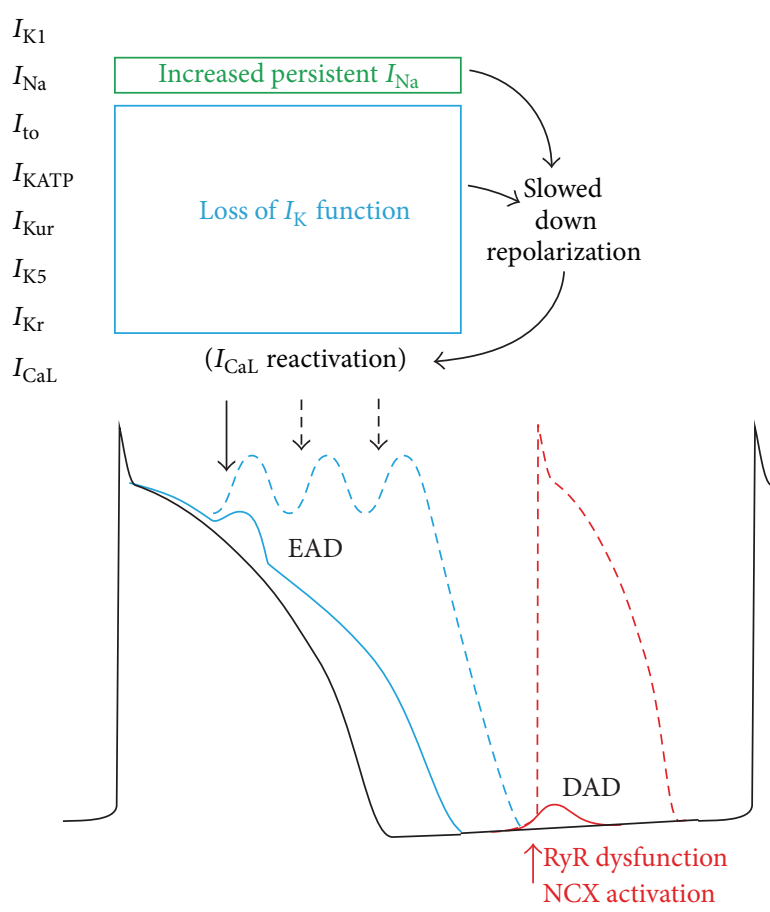

(b)

FIGURE 2: Illustrative diagram of a typical human left atrial AP with associated currents shown above (a). Electrical remodeling can result in abnormal APs (b). $I_{\mathrm{CaL}}$ reactivation (blue) can cause early-after depolarization (EAD) that may result in repetitive EAD. Alternatively spontaneous ryanodine receptor (RYR) release or $\mathrm{Na}^{+} / \mathrm{Ca}^{2+}$ exchanger activation (NCX) (red) would result in delayed-after depolarization (DAD) (unpublished).

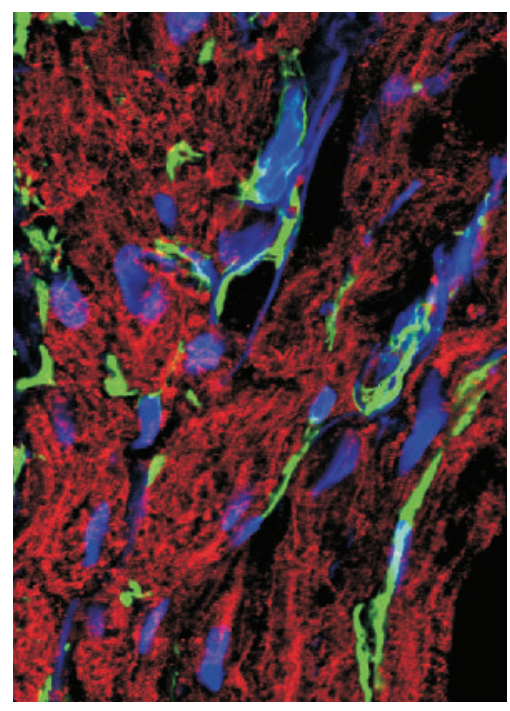

(a)

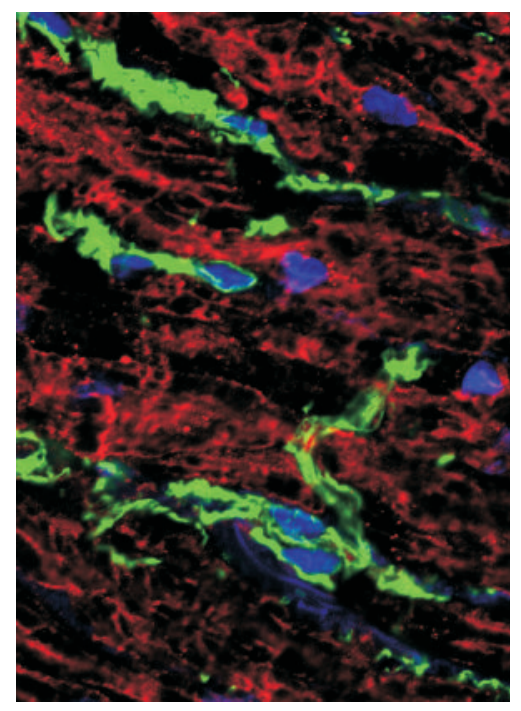

(b)

FIGURE 3: Staining of the left atrial appendage demonstrating fibrosis stained with vimentin (green) in a patient in sinus rhythm (a) and AF (b) (unpublished).

\section{Biomarkers for Paroxysmal Atrial Fibrillation}

5.1. Electrophysiological Biomarkers. A number of studies have shown that simple resting ECG parameters are highly predictive of PAF. "P-maximum" is the maximum duration from the onset to the end of the P-wave deflection from all 12 ECG leads. This is seen as a marker of prolonged atrial conduction time, a hallmark of patients with AF. "P wave dispersion," the difference between the maximum and minimum 


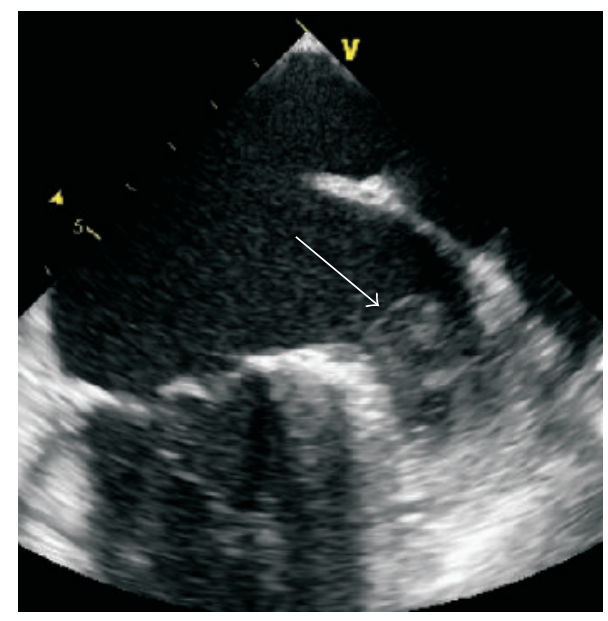

FIGURE 4: Thrombus visualized in the left atrial appendage is marked with an arrow (unpublished).

P-wave duration in any of the 12 leads, signifies nonuniform atrial conductivity. Both parameters are representative of the underlying atrial remodelling occurring in AF and previously alluded to. One study showed that P-maximum and P-wave dispersion were highly predictive of previous episodes of PAF. A P-maximum of at least $110 \mathrm{~ms}$ had a sensitivity of $88 \%$ and specificity of $75 \%$ and a P-wave dispersion of at least $40 \mathrm{~ms}$ yielded a sensitivity of $83 \%$ and specificity of $85 \%$ for PAF [26]. These findings have been substantiated by another study showing that P-wave dispersion was an independent predictor of PAF in stroke patients. In this population a Pwave dispersion $57.5 \mathrm{~ms}$ and above predicted PAF with a sensitivity and specificity of $80 \%$ and $73 \%$, respectively [27].

Likewise a J-shaped relationship was shown between the QTc interval and risk of incident AF in a large primary care population. QTc intervals at the 99th percentile and above $(\geq 464 \mathrm{~ms})$ resulted in an overall hazard ratio of 1.44 for all AF subtypes. Additionally an even more powerful relationship was seen in lone AF with a QTc interval $\geq 458 \mathrm{~ms}$ demonstrating a hazard ratio of 2.32. Conversely a QTc $\leq 372 \mathrm{~ms}$ was associated with a hazard ratio of 1.45 [28]. More specific to PAF, another group have shown that a prolonged QTc interval is an independent predictor of PAF in patients presenting with ischaemic stroke. In this instance a QTc threshold $\geq 438 \mathrm{~ms}$ generated a sensitivity of $59.4 \%$ and specificity of $83.7 \%$ [29]. It has been proposed that these associations may be on account of universal expression of ion channels in both atrial and ventricular tissue.

Atrial premature beats (APBs) precede PAF in a significant proportion of AF episodes and are a feature of enhanced automaticity, a recognised mechanism for AF (Figure 5). In one study monitoring 33 patients with documented PAF and 297 total episodes of AF, the arrhythmia was initiated by APBs in $93 \%$ of cases [30]. Consequently frequent APBs have been suggested as a marker of predisposition to PAF. One study investigating 98 stroke patients with transtelephonic monitoring determined that at least 100 APBs on a 24-hour Holter monitor equated to an odds ratio of 11 for the subsequent development of PAF after one month [31].

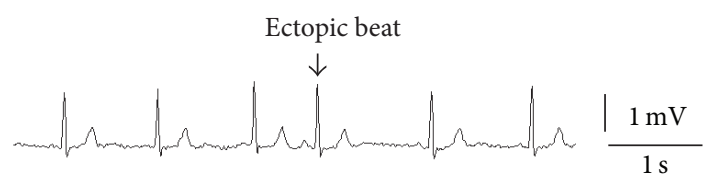

Figure 5: An example of an atrial premature beat is marked (unpublished).

In another cohort of stroke patients, greater than 4 APBs per hour during an initial 24-hour Holter monitor equated to subsequent development of PAF in $19.6 \%$, compared to $2.8 \%$ in those with 4 APBs per hour or less [32]. Using a similar study design, Wallmann et al. also deduced that frequent APBs were an independent risk factor for PAF in stroke patients. Significantly more of those patients with frequent APBs (defined as at least 70 in 24 hours) developed PAF at 22.4 months compared with those with infrequent APBs $(33 \%$ versus 5\%) [33].

5.2. Molecular Biomarkers. Previously validated biomarkers, currently used in standard clinical practice to diagnose cardiac failure and myocardial infarction, have also been tested in the setting of AF. Numerous studies have shown an association between AF and brain natriuretic peptide (BNP) with persistently higher plasma levels than in healthy matched controls and a reduction to that of control subjects following successful restoration of sinus rhythm [34]. It has been proposed that the main source of BNP release in AF is the atrium as a result of pressure and volume overload. It remains to be established however whether BNP is merely a marker of atrial dysfunction or active in the underlying pathological process [35]. With a particular focus on PAF, several studies have shown a relationship between natriuretic peptides and diagnosis of this arrhythmia. One study showed higher levels of plasma BNP in patients with lone PAF compared to ageand sex-matched controls [36]. A further study confirmed significantly raised N-terminal of prohormone brain natriuretic peptide (NT-proBNP, a peptide cleaved from pro$\mathrm{BNP}$ to release BNP) in contrast to matched controls. Yet a similar relationship was not demonstrated for pro-ANP (atrial natriuretic peptide) in this group [37]. Likewise in the Find-AF study, BNP levels were significantly higher in those cryptogenic stroke patients with confirmed PAF than those without [38]. A similar prospective study found that a BNP over $140 \mathrm{pg} / \mathrm{mL}$ had a sensitivity and specificity of $77.6 \%$ and $84.8 \%$, respectively, for cardioembolic stroke [39]. Likewise NT-proBNP levels over $265.5 \mathrm{pg} / \mathrm{mL}$ conferred a sensitivity $100 \%$ and specificity $70.5 \%$ for PAF [40]. Similarly troponin, another established cardiac biomarker, is increased in PAF subjects relative to their controls. Elevation of troponin levels in cryptogenic stroke patients independently predicted new onset AF during 24-hour Holter monitoring [41]. This finding was also confirmed in a similar retrospective study [42].

Markers of inflammation, implicated in the pathogenesis of AF, have yielded mixed results. Raised serum IL-18 was significantly associated with both PAF and persistent AF with a twofold concentration in all AF subtypes compared 
to controls [43]. Another study confirmed that patients with PAF had significantly higher levels of CRP (C-reactive protein) than their controls, in a graded fashion according to arrhythmia burden [44]. Similarly a raised white blood cell count predisposed to incident AF after 5 years in the Framingham Study [45]. In stark contrast another study demonstrated no difference in the inflammatory markers CRP, IL-6, and IL- 8 between PAF patients and their controls when attending for radiofrequency ablation [46]. Glucose haemostasis and lipid metabolism also appear to be associated with the development of AF. In the Atherosclerosis Risk in Communities (ARIC) cohort a positive linear relationship was seen between haemoglobin Alc (HbAlc) and incident $\mathrm{AF}$ in patients both with and without type 2 diabetes [47]. Interestingly in the Women's Health Study however, although HbAlc was positively correlated with the incidence of nonparoxysmal AF, it was inversely related to PAF [48]. Additionally the ARIC study demonstrated that both higher LDL and total cholesterol resulted in a lower incidence of all AF subtypes [49]. This, somewhat unexpected, inverse relationship was replicated by another group [50] yet an explanation for this repeated finding is not clear.

Parameters reflecting thrombogenesis, the source of cardioembolism and stroke in PAF, have also been investigated. For example, plasma von Willebrand factor and fibrinogen were significantly elevated in patients with PAF compared to their matched controls [51]. Similarly fibrinogen and fibrin D-dimer were significantly increased in PAF relative to ageand sex-matched controls [52]. Additionally regulators of extracellular turnover, the culprits of the previously described fibrotic remodelling, were all increased in lone PAF subjects compared to their controls. These markers include CICP (C-terminal propeptide of collagen type-I), CITP (Cterminal telopeptide of collagen type-I), MMP-1 (matrix metalloproteinase-1), and TIMP-1 (tissue inhibitor of matrix metalloproteinase) [53]. Likewise CITP was also raised in PAF cases compared to healthy subjects in another study [54]. Both of these studies were limited by a younger control group however, introducing potential confounding.

A genetic predisposition has been shown to contribute to the development of AF. For example, the Framingham Study showed a heightened risk of AF in the children of a parent with AF, independent of other risk factors (odds ratio of 1.85) [55]. Genetic analysis of over 14,000 European and Japanese individuals with a history of AF noted susceptibility signals on chromosome $4 \mathrm{q} 25$, upstream of PTIX2, a gene previously implicated in AF [56]. Furthermore six further susceptibility loci (1q24, 7q31, 14q23, 9q22, 15q24, and $10 \mathrm{q} 22)$ were identified in a meta-analysis comprising over 10,000 individuals with AF of European decent [57]. These findings prompted the Women's Health Study to validate a prediction score for incident AF incorporating 12 singlenucleotide polymorphisms in nine loci. Genetic risk markers were shown to improve a conventional clinical risk score from a C-statistic of 0.718 to $0.741(P=0.001)$ [58]. Whether genetic markers will aid in the diagnosis of PAF in particular is, as yet, to be definitively answered. There is however promising research suggesting that microRNAs, stable noncoding RNAs (ribonucleic acid) found in serum and plasma which modulate RNA transcription, may play such a role. There is a relative paucity of research in this area to date and studies investigating a link to PAF are even scarcer. However promisingly, several preliminary studies have shown that plasma miRNA-150 expression, already implicated in the regulation of genes associated with atrial remodeling, is significantly reduced in individuals with PAF $[59,60]$.

5.3. Morphological Biomarkers. It is widely recognised that LA size is a powerful predictor of cardiovascular mortality, conferring an independent 2.3-fold excess risk of cardiovascular mortality at 13 years [61]. Specific to AF, each increase in LA diameter by $5 \mathrm{~mm}$ increased the risk of new AF by 39\% in the Framingham Heart Study [62]. Likewise subjects with PAF had increased LA size relative to healthy controls with an inferosuperior dimension of at least $50 \mathrm{~mm}$, yielding a sensitivity and specificity for PAF of $66 \%$ and $80 \%$, respectively [63]. These observations are a direct result of the structural remodelling occurring in the LA in the course of the disease process.

With the advent of increasingly sophisticated techniques to assess the LA, echocardiographic indices of function are now widely thought to be more robust predictors of outcomes than size alone $[64,65]$. Methods to assess LA function include Doppler analysis of transmitral flow and tissue Doppler assessment of LA myocardial velocities. Not only have alterations in LA function been shown to be a hallmark for PAF, but also they may predict the onset of AF following cardiothoracic surgery, cardioversion, and ablation, as well as being an independent marker of thromboembolic risk [66-69]. Variables of LA function, as assessed using transoesophageal echocardiography (TOE), were impaired in PAF patients following stroke. LAA peak velocity was markedly reduced in the PAF group compared to the control group. Additionally LA spontaneous echo contrast, graded from 0 (none) to $4+$ (severe), was higher in the PAF group. Both of these measurements reflect atrial stasis and were more powerful predictors of PAF than LA dimension alone [70].

These results have been replicated using transthoracic echocardiography where, LA function as assessed by myocardial Doppler, is thought to predict PAF more precisely than LA dimensions. Toh et al. used a novel marker of both LA size combined with LA pump function $\left[\mathrm{LAVI} / \mathrm{a}^{\prime}\right]$ to discriminate between patients with PAF in a group of hypertensive patients. LAVI (left atrial volume index) is calculated from the LA volume corrected for body surface area. Left atrial velocity in late diastole, or $\mathrm{a}^{\prime}$, is derived from myocardial tissue Doppler measured from the mitral annulus (Figure 6). In this population a LAVI/a' threshold of 2.7 conferred a sensitivity $82 \%$ and specificity $91 \%$ for PAF. [71]. The Find-AF group used the same method to distinguish between stroke or TIA patients with underlying PAF. It was deduced that LAVI $/ \mathrm{a}^{\prime}$ of 2.3 had a $93 \%$ sensitivity and $55.8 \%$ specificity. Again this parameter predicted PAF more powerfully than measures of LA size, including LA diameter and LAVI [72].

Mitral valve disease is commonly associated with a concurrent diagnosis AF, attributed to atrial remodeling and 


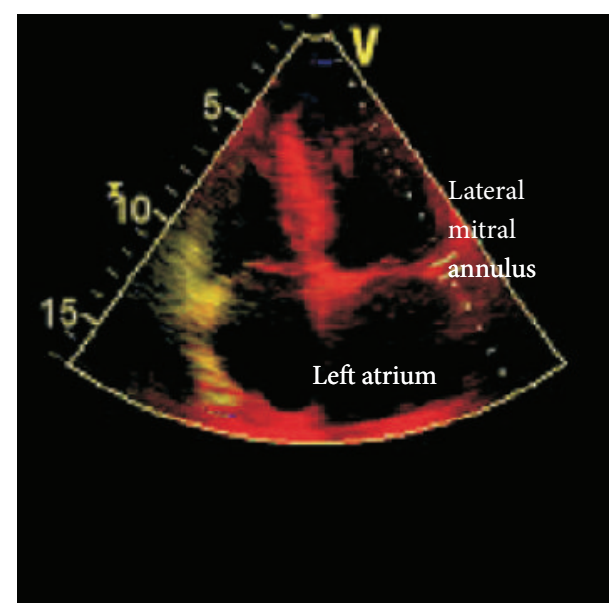

(a)

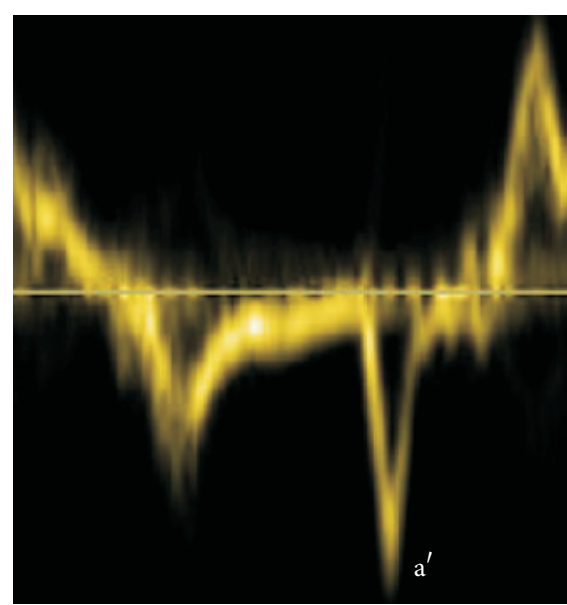

(b)

FIgURE 6: Measurement of left atrial velocity using tissue Doppler. The left image demonstrates the position of the probe at the lateral mitral annulus. The right image represents the velocities generated during diastole with the atrial component (termed a') marked (unpublished).

dilatation as a result of volume and pressure overload. For example, over $40 \%$ of individuals with rheumatic mitral valve disease and between 44 and $48 \%$ of those with degenerative mitral regurgitation (MR) develop AF in the long-term $[73,74]$. One observational study has proposed a form of "functional MR" as a direct consequent of AF, possibly induced by atrial wall dyssynchrony, which improves following restoration of sinus rhythm [75]. As a predictive tool, one group proposed that, in patients with at least moderate $M R$, the rate of left ventricular pressure rise in early systole $(d P / d t$ max) independently predicted new onset AF or ischaemic stroke. The study was significantly limited by a small sample size with only 9 patients having this combined end point [76]. To our knowledge no large studies assessing the use of $\mathrm{MR}$ as a diagnostic marker for PAF specifically have been undertaken to date.

\section{Conclusions}

Despite several decades of plausible research suggesting that biomarkers might be used to improve the diagnosis of PAF (summarised in Table 1) these techniques have not as yet entered clinic practice. This begs the question why this might be the case.

There appears to have been a shift in clinicians' and scientists' attitudes to AF in recent years and PAF is no longer seen as a benign entity. This and the impending "AF epidemic" have highlighted the importance of optimal arrhythmia detection. This had been somewhat neglected previously with the 24-hour Holter monitor remaining commonplace as a first-line investigation. Critically the lack of a universal definition for PAF has hindered research in the field. AF research is riddled with conflicting definitions primarily dependent on the duration of the arrhythmia and tendency to self-terminate. Furthermore a lack of consensus may be due to oversimplified classification systems. AF is a heterogeneous condition likely to represent a number of underlying pathologies and a lack of aetiological classification may have hindered the quest for improved diagnostic markers.

The majority of the literature in this review suggests that biomarkers might be a valuable addition to current investigational techniques. However these findings are admittedly subject to inherent publication bias. Furthermore recurrent methodological flaws are encountered. Many of the papers published in the area consist of small populations. Additionally a number of groups relied on self-reported AF as opposed to objective screening. Crucially many of the control groups were younger than those with confirmed PAF. Given that age is an independent risk factor for AF and with age comes increasing comorbidity, this introduces confounding. Lastly a number of the techniques described are time-consuming and some echocardiographic techniques are hindered by difficult patient anatomy.

In summary it remains an open question if biomarkers will add to conventional diagnostic techniques for PAF. If the role of biomarkers is to be established then future research requires refinement. We propose that an accurate future biomarker is likely to feature a combination of electrophysiological, molecular, and morphological indices therefore encompassing the heterogeneity seen in this condition. A reliable diagnostic marker to improve the diagnosis of undetected PAF and ultimately reduce risk of consequent thromboembolism certainly warrants further investigation.

\section{Conflict of Interests}

The authors declare that there is no conflict of interests regarding the publication of this paper.

\section{Acknowledgments}

The authors would like to thank the Heart and Stroke Trust Endeavour (HASTE) for supporting their work. They are also grateful to Dr. Krystyna Darasz for supplying Figure 4. 


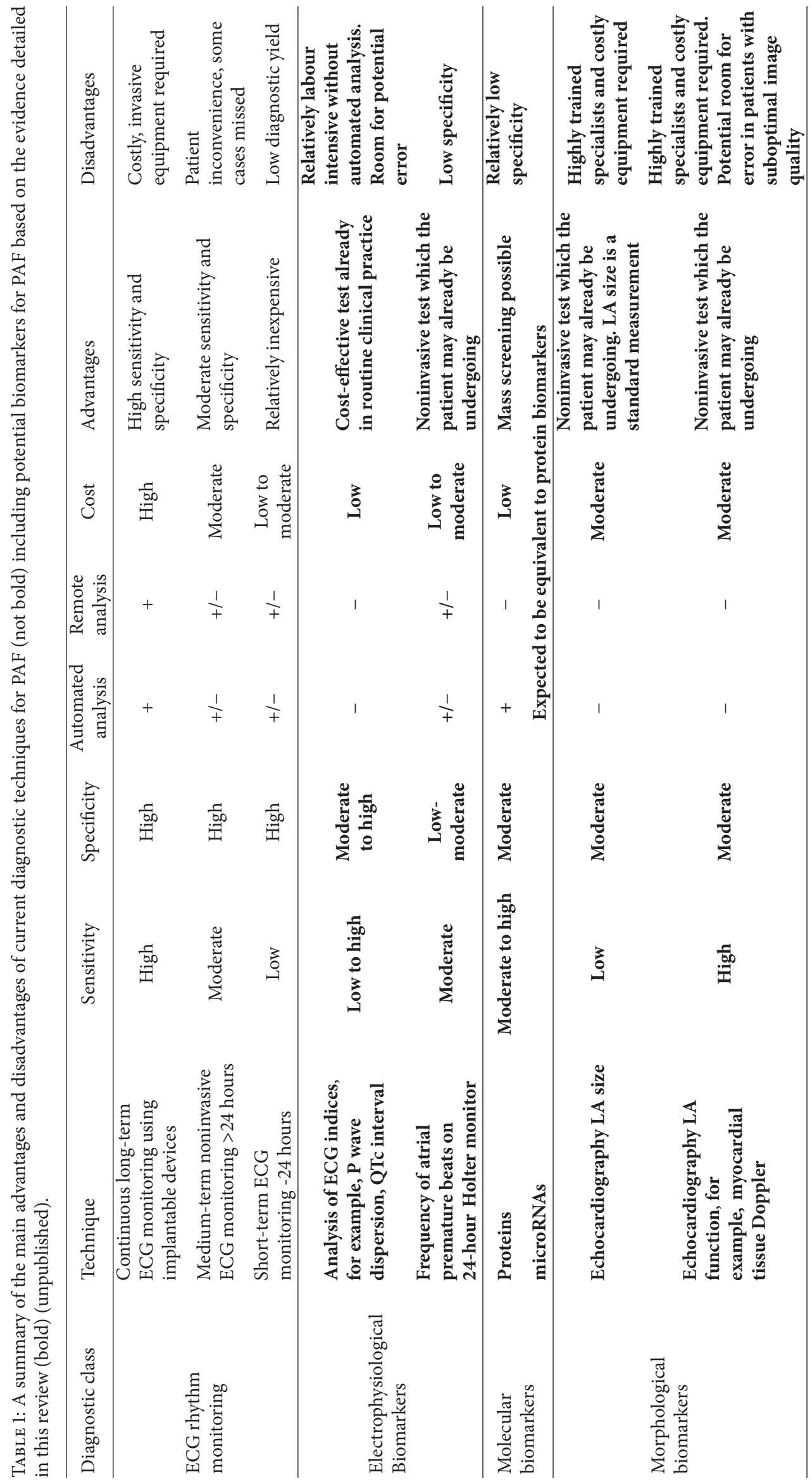




\section{References}

[1] D. M. Lloyd-Jones, T. J. Wang, E. P. Leip et al., "Lifetime risk for development of atrial fibrillation: the framingham heart study," Circulation, vol. 110, no. 9, pp. 1042-1046, 2004.

[2] Y. Miyasaka, M. E. Barnes, B. J. Gersh et al., "Secular trends in incidence of atrial fibrillation in Olmsted County, Minnesota, 1980 to 2000, and implications on the projections for future prevalence," Circulation, vol. 114, no. 2, pp. 119-125, 2006.

[3] D. A. Dulli, H. Stanko, and R. L. Levine, "Atrial fibrillation is associated with severe acute ischemic stroke," Neuroepidemiology, vol. 22, no. 2, pp. 118-123, 2003.

[4] H.-J. Lin, P. A. Wolf, M. Kelly-Hayes et al., "Stroke severity in atrial fibrillation: the Framingham study," Stroke, vol. 27, no. 10, pp. 1760-1764, 1996.

[5] J. L. Clua-Espuny, I. Lechuga-Duran, R. Bosch-Princep et al., "Prevalence of undiagnosed atrial fibrillation and of that not being treated with anticoagulant drugs: the AFABE study," Revista Española de Cardiología, vol. 66, no. 7, pp. 545-552, 2013.

[6] R. C. S. Seet, P. A. Friedman, and A. A. Rabinstein, "Prolonged rhythm monitoring for the detection of occult paroxysmal atrial fibrillation in ischemic stroke of unknown cause," Circulation, vol. 124, no. 4, pp. 477-486, 2011.

[7] V. Fuster, L. E. Rydén, D. S. Cannom et al., "ACC/AHA/ESC 2006 Guidelines for the Management of Patients with Atrial Fibrillation: a report of the American College of Cardiology/American Heart Association Task Force on Practice Guidelines and the European Society of Cardiology Committee for Practice Guidelines," Circulation, vol. 114, no. 7, pp. e257-e354, 2006.

[8] M. Khan and D. J. Miller, "Detection of paroxysmal atrial fibrillation in stroke/Tia patients," Stroke Research and Treatment, vol. 2013, Article ID 840265, 5 pages, 2013.

[9] R. G. Hart, L. A. Pearce, R. M. Rothbart, J. H. McAnulty, R. W. Asinger, and J. L. Halperin, "Stroke with intermittent atrial fibrillation: incidence and predictors during aspirin therapy," Journal of the American College of Cardiology, vol. 35, no. 1, pp. 183-187, 2000.

[10] R. L. Page, W. E. Wilkinson, W. K. Clair, E. A. McCarthy, and E. L. C. Pritchett, "Asymptomatic arrhythmias in patients with symptomatic paroxysmal atrial fibrillation and paroxysmal supraventricular tachycardia," Circulation, vol. 89, no. 1, pp. 224-227, 1994.

[11] N. Voigt, J. Heijman, Q. Wang et al., "Cellular and molecular mechanisms of atrial arrhythmogenesis in patients with paroxysmal atrial fibrillation," Circulation, vol. 129, no. 2, pp. 145-156, 2014.

[12] Y.-H. Yeh, R. Wakili, X.-Y. Qi et al., "Calcium-handling abnormalities underlying atrial arrhythmogenesis and contractile dysfunction in dogs with congestive heart failure," Circulation: Arrhythmia and Electrophysiology, vol. 1, no. 2, pp. 93-102, 2008.

[13] J. Monigatti-Tenkorang, F. Jousset, P. Pascale et al., "Intermittent atrial tachycardia promotes repolarization alternans and conduction slowing during rapid rates, and increases susceptibility to atrial fibrillation in a free-behaving sheep model," Journal of Cardiovascular Electrophysiology, vol. 25, no. 4, pp. 418-427, 2014.

[14] A. Goette, T. Staack, C. Röcken et al., "Increased expression of extracellular signal-regulated kinase and angiotensinconverting enzyme in human atria during atrial fibrillation," Journal of the American College of Cardiology, vol. 35, no. 6, pp. 1669-1677, 2000.
[15] R. Zhang, Y. Y. Zhang, X. R. Huang et al., "C-reactive protein promotes cardiac fibrosis and inflammation in angiotensin IIinduced hypertensive cardiac disease," Hypertension, vol. 55, no. 4, pp. 953-960, 2010.

[16] Y. Guo, G. Y. H. Lip, and S. Apostolakis, "Inflammation in atrial fibrillation," Journal of the American College of Cardiology, vol. 60, no. 22, pp. 2263-2270, 2012.

[17] J. L. Blackshear and J. A. Odell, "Appendage obliteration to reduce stroke in cardiac surgical patients with atrial fibrillation," Annals of Thoracic Surgery, vol. 61, no. 2, pp. 755-759, 1996.

[18] T. Watson, E. Shantsila, and G. Y. Lip, "Mechanisms of thrombogenesis in atrial fibrillation: virchow's triad revisited," The Lancet, vol. 373, no. 9658, pp. 155-166, 2009.

[19] T. Sanna, H. C. C. Diener, R. S. Passman et al., "Cryptogenic stroke and underlying atrial fibrillation," The New England Journal of Medicine, vol. 370, no. 26, pp. 2478-2486, 2014.

[20] D. J. Gladstone, M. Spring, P. Dorian et al., "Atrial fibrillation in patients with cryptogenic stroke," The New England Journal of Medicine, vol. 370, no. 26, pp. 2467-2477, 2014.

[21] R. B. Schnabel, L. M. Sullivan, D. Levy et al., "Development of a risk score for atrial fibrillation (Framingham Heart Study): a community-based cohort study," The Lancet, vol. 373, no. 9665, pp. 739-745, 2009.

[22] A. Mosterd and A. W. Hoes, "Clinical epidemiology of heart failure," Heart, vol. 93, no. 9, pp. 1137-1146, 2007.

[23] Biomarkers Definitions Working Group, "Biomarkers and surrogate endpoints: preferred definitions and conceptual framework," Clinical Pharmacology and Therapeutics, vol. 69, no. 3, pp. 89-95, 2001.

[24] R. P. Martins, K. Kaur, E. Hwang et al., "Dominant frequency increase rate predicts transition from paroxysmal to long-term persistent atrial fibrillation," Circulation, vol. 129, no. 14, pp. 1472-1482, 2014.

[25] Z. Hijazi, J. Oldgren, U. Andersson et al., "Cardiac biomarkers are associated with an increased risk of stroke and death in patients with atrial fibrillation: a randomized evaluation of long-term anticoagulation therapy (RE-LY) substudy," Circulation, vol. 125, no. 13, pp. 1605-1616, 2012.

[26] P. E. Dilaveris, E. J. Gialafos, S. K. Sideris et al., "Simple electrocardiographic markers for the prediction of paroxysmal idiopathic atrial fibrillation," The American Heart Journal, vol. 135, no. 5, par 1, pp. 733-738, 1998.

[27] U. Dogan, E. A. Dogan, M. Tekinalp et al., "P-wave dispersion for predicting paroxysmal atrial fibrillation in acute ischemic stroke," International Journal of Medical Sciences, vol. 9, no. 1, pp. 108-114, 2012.

[28] J. B. Nielsen, C. Graff, A. Pietersen et al., "J-shaped association between QTc interval duration and the risk of atrial fibrillation: results from the copenhagen ECG study," Journal of the American College of Cardiology, vol. 61, no. 25, pp. 2557-2564, 2013.

[29] T. Hoshino, T. Nagao, T. Shiga et al., "Prolonged QTc interval predicts poststroke paroxysmal atrial fibrillation," Stroke, vol. 46, pp. 71-76, 2015.

[30] C. Kolb, S. Nürnberger, G. Ndrepepa, B. Zrenner, A. Schömig, and C. Schmitt, "Modes of initiation of paroxysmal atrial fibrillation from analysis of spontaneously occurring episodes using a 12-lead Holter monitoring system," The American Journal of Cardiology, vol. 88, no. 8, pp. 853-857, 2001.

[31] N. Gaillard, S. Deltour, B. Vilotijevic et al., "Detection of paroxysmal atrial fibrillation with transtelephonic EKG in TIA or stroke patients," Neurology, vol. 74, no. 21, pp. 1666-1670, 2010. 
[32] M. Weber-Krüger, K. Gröschel, M. Mende et al., "Excessive supraventricular ectopic activity is indicative of paroxysmal atrial fibrillation in patients with cerebral ischemia," PLoS ONE, vol. 8, no. 6, Article ID e67602, 2013.

[33] D. Wallmann, D. Tüller, K. Wustmann et al., "Frequent atrial premature beats predict paroxysmal atrial fibrillation in stroke patients: an opportunity for a new diagnostic strategy," Stroke, vol. 38, no. 8, pp. 2292-2294, 2007.

[34] B. Wozakowska-Kapłon, "Effect of sinus rhythm restoration on plasma brain natriuretic peptide in patients with atrial fibrillation," The American Journal of Cardiology, vol. 93, no. 12, pp. 1555-1558, 2004.

[35] S.-I. Inoue, Y. Murakami, K. Sano, H. Katoh, and T. Shimada, "Atrium as a source of brain natriuretic polypeptide in patients with atrial fibrillation," Journal of Cardiac Failure, vol. 6, no. 2, pp. 92-96, 2000.

[36] J. Li and L. Wang, "B-type natriuretic peptide levels in patients with paroxysmal lone atrial fibrillation," Heart and Vessels, vol. 21, no. 3, pp. 137-140, 2006.

[37] P. T. Ellinor, A. F. Low, K. K. Patton, M. A. Shea, and C. A. MacRae, "Discordant atrial natriuretic peptide and brain natriuretic peptide levels in lone atrial fibrillation," Journal of the American College of Cardiology, vol. 45, no. 1, pp. 82-86, 2005.

[38] R. Wachter, R. Lahno, B. Haase et al., "Natriuretic peptides for the detection of paroxysmal atrial fibrillation in patients with cerebral ischemia-the find-AF study," PLoS ONE, vol. 7, no. 4, Article ID e34351, 2012.

[39] K. Sakai, K. Shibazaki, K. Kimura et al., "Brain natriuretic peptide as a predictor of cardioembolism in acute ischemic stroke patients: brain natriuretic peptide stroke prospective study," European Neurology, vol. 69, no. 4, pp. 246-251, 2013.

[40] A. C. Fonseca, D. Brito, T. Pinho e Melo et al., "N-terminal probrain natriuretic peptide shows diagnostic accuracy for detecting atrial fibrillation in cryptogenic stroke patients," International Journal of Stroke, vol. 9, no. 4, pp. 419-425, 2014.

[41] I. Beaulieu-Boire, N. Leblanc, L. Berger, and J.-M. M. Boulanger, "Troponin elevation predicts atrial fibrillation in patients with stroke or transient ischemic attack," Journal of Stroke and Cerebrovascular Diseases, vol. 22, no. 7, pp. 978-983, 2013.

[42] J.-M. M. Bugnicourt, V. Rogez, M.-P. P. Guillaumont, J.-C. C. Rogez, S. Canaple, and O. Godefroy, "Troponin levels help predict new-onset atrial fibrillation in ischaemic stroke patients: a retrospective study," European Neurology, vol. 63, no. 1, pp. 2428, 2010.

[43] Y. Luan, Y. Guo, S. Li et al., "Interleukin-18 among atrial fibrillation patients in the absence of structural heart disease," Europace, vol. 12, no. 12, pp. 1713-1718, 2010.

[44] M. K. Chung, D. O. Martin, D. Sprecher et al., "C-reactive protein elevation in patients with atrial arrhythmias: inflammatory mechanisms and persistence of atrial fibrillation," Circulation, vol. 104, no. 24, pp. 2886-2891, 2001.

[45] M. Rienstra, J. X. Sun, J. W. Magnani et al., "White blood cell count and risk of incident atrial fibrillation (from the framingham heart study)," The American Journal of Cardiology, vol. 109, no. 4, pp. 533-537, 2012.

[46] I. Liuba, H. Ahlmroth, L. Jonasson et al., "Source of inflammatory markers in patients with atrial fibrillation," Europace, vol. 10, no. 7, pp. 848-853, 2008.

[47] R. R. Huxley, A. Alonso, F. L. Lopez et al., “Type 2 diabetes, glucose homeostasis and incident atrial fibrillation: the Atherosclerosis risk in communities study," Heart, vol. 98, no. 2, pp. 133-138, 2012.
[48] R. K. Sandhu, D. Conen, U. B. Tedrow et al., "Predisposing factors associated with development of persistent compared with paroxysmal atrial fibrillation," Journal of the American Heart Association, vol. 3, no. 3, Article ID e000916, 2014.

[49] F. L. Lopez, S. K. Agarwal, R. F. MacLehose et al., "Blood lipid levels, lipid-lowering medications, and the incidence of atrial fibrillation : the Atherosclerosis Risk in Communities (ARIC) study," Circulation: Arrhythmia and Electrophysiology, vol. 5, no. 1, pp. 155-162, 2012.

[50] B. M. Psaty, T. A. Manolio, L. H. Kuller et al., "Incidence of and risk factors for atrial fibrillation in older adults," Circulation, vol. 96, no. 7, pp. 2455-2461, 1997.

[51] F. L. Li-Saw-Hee, A. D. Blann, D. Gurney, and G. Y. H. Lip, "Plasma von Willebrand factor, fibrinogen and soluble Pselectin levels in paroxysmal, persistent and permanent atrial fibrillation: effects of cardioversion and return of left atrial function," European Heart Journal, vol. 22, no. 18, pp. 1741-1747, 2001.

[52] G. Y. H. Lip, G. D. O. Lowe, A. Rumley, and F. G. Dunn, "Fibrinogen and fibrin $\mathrm{D}$-dimer levels in paroxysmal atrial fibrillation: evidence for intermediate elevated levels of intravascular thrombogenesis," American Heart Journal, vol. 131, no. 4, pp. 724-730, 1996.

[53] E. M. Kallergis, E. G. Manios, E. M. Kanoupakis et al., "Extracellular matrix alterations in patients with paroxysmal and persistent atrial fibrillation: biochemical assessment of collagen type-I turnover," Journal of the American College of Cardiology, vol. 52, no. 3, pp. 211-215, 2008.

[54] M. Fujita, X. W. Cheng, Y. Inden et al., "Mechanisms with clinical implications for atrial fibrillation-associated remodeling: cathepsin $\mathrm{K}$ expression, regulation, and therapeutic target and biomarker," Journal of the American Heart Association, vol. 2, Article ID e000503, 2013.

[55] C. S. Fox, H. Parise, R. B. D’Agostino et al., "Parental atrial fibrillation as a risk factor for atrial fibrillation in offspring," Journal of the American Medical Association, vol. 291, no. 23, pp. 2851-2855, 2004.

[56] S. A. Lubitz, K. L. Lunetta, H. Lin et al., "Novel genetic markers associated with atrial fibrillation risk in Europeans and Japanese," Journal of the American College of Cardiology, vol. 63, no. 12, pp. 1200-1210, 2014.

[57] P. T. Ellinor, K. L. Lunetta, C. M. Albert et al., "Meta-analysis identifies six new susceptibility loci for atrial fibrillation," Nature Genetics, vol. 44, no. 6, pp. 670-675, 2012.

[58] B. M. Everett, N. R. Cook, D. Conen, D. I. Chasman, P. M. Ridker, and C. M. Albert, "Novel genetic markers improve measures of atrial fibrillation risk prediction," European Heart Journal, vol. 34, no. 29, pp. 2243-2251, 2013.

[59] Z. Liu, C. Zhou, Y. Liu et al., "The expression levels of plasma microRNAs in atrial fibrillation patients," PLoS ONE, vol. 7, no. 9, Article ID e44906, 2012.

[60] D. D. McManus, K. Tanriverdi, H. Lin et al., "Plasma microRNAs are associated with atrial fibrillation and change after catheter ablation (the miRhythm study)," Heart Rhythm, vol. 12, no. 1, pp. 3-10, 2015.

[61] J. A. Laukkanen, S. Kurl, J. Eränen, M. Huttunen, and J. T. Salonen, "Left atrium size and the risk of cardiovascular death in middle-aged men," Archives of Internal Medicine, vol. 165, no. 15, pp. 1788-1793, 2005.

[62] S. M. Vaziri, M. G. Larson, E. J. Benjamin, and D. Levy, "Echocardiographic predictors of nonrheumatic atrial 
fibrillation. The Framingham Heart Study," Circulation, vol. 89, no. 2, pp. 724-730, 1994.

[63] R. S. Phang, S. M. Isserman, D. Karia et al., "Echocardiographic evidence of left atrial abnormality in young patients with lone paroxysmal atrial fibrillation," The American Journal of Cardiology, vol. 94, no. 4, pp. 511-513, 2004.

[64] W. P. Abhayaratna, K. Fatema, M. E. Barnes et al., "Left atrial reservoir function as a potent marker for first atrial fibrillation or flutter in persons $>$ or $=65$ years of age," The American Journal of Cardiology, vol. 101, no. 11, pp. 1626-1629, 2008.

[65] J. H. Yoon, J. Moon, H. M. Chung et al., "Left atrial function assessed by doppler echocardiography rather than left atrial volume predicts recurrence in patients with paroxysmal atrial fibrillation," Clinical Cardiology, vol. 36, no. 4, pp. 235-240, 2013.

[66] J. M. Leung, W. H. Bellows, and N. B. Schiller, "Impairment of left atrial function predicts post-operative atrial fibrillation after coronary artery bypass graft surgery," European Heart Journal, vol. 25, no. 20, pp. 1836-1844, 2004.

[67] G. di Salvo, P. Caso, R. Lo Piccolo et al., "Atrial myocardial deformation properties predict maintenance of sinus rhythm after external cardioversion of recent-onset lone atrial fibrillation: a color Doppler myocardial imaging and transthoracic and transesophageal echocardiographic study," Circulation, vol. 112, no. 3, pp. 387-395, 2005.

[68] C. Schneider, R. Malisius, K. Krause et al., "Strain rate imaging for functional quantification of the left atrium: atrial deformation predicts the maintenance of sinus rhythm after catheter ablation of atrial fibrillation," European Heart Journal, vol. 29, no. 11, pp. 1397-1409, 2008.

[69] M. Zabalgoitia, J. L. Halperin, L. A. Pearce, J. L. Blackshear, R. W. Asinger, and R. G. Hart, "Transesophageal echocardiographic correlates of clinical risk of thromboembolism in nonvalvular atrial fibrillation. Stroke Prevention in Atrial Fibrillation III Investigators," Journal of the American College of Cardiology, vol. 31, no. 7, pp. 1622-1626, 1998.

[70] Y. Taguchi, S. Takashima, T. Hirai et al., "Significant impairment of left atrial function in patients with cardioembolic stroke caused by paroxysmal atrial fibrillation," Internal Medicine, vol. 49, no. 16, pp. 1727-1732, 2010.

[71] N. Toh, H. Kanzaki, S. Nakatani et al., "Left atrial volume combined with atrial pump function identifies hypertensive patients with a history of paroxysmal atrial fibrillation," Hypertension, vol. 55, no. 5, pp. 1150-1156, 2010.

[72] R. Stahrenberg, F. Edelmann, B. Haase et al., "Transthoracic echocardiography to rule out paroxysmal atrial fibrillation as a cause of stroke or transient ischemic attack," Stroke, vol. 42, no. 12, pp. 3643-3645, 2011.

[73] A. Selzer and K. E. Cohn, "Natural history of mitral stenosis: a review," Circulation, vol. 45, no. 4, pp. 878-890, 1972.

[74] F. Grigioni, J.-F. Avierinos, L. H. Ling et al., "Atrial fibrillation complicating the course of degenerative mitral regurgitation: determinants and long-term outcome," Journal of the American College of Cardiology, vol. 40, no. 1, pp. 84-92, 2002.

[75] Z. M. Gertz, A. Raina, L. Saghy et al., "Evidence of atrial functional mitral regurgitation due to atrial fibrillation: reversal with arrhythmia control," Journal of the American College of Cardiology, vol. 58, no. 14, pp. 1474-1481, 2011.

[76] J. Yi, D. Lee, E. J. Cho, H. Jeon, H. Jung, and H. Youn, “Dopplerderived left ventricular negative $\mathrm{dP} / \mathrm{dt}$ as a predictor of atrial fibrillation or ischamic stroke in patients with degenerative mitral regurgitation and normal ejection fraction," Echocardiography, vol. 31, no. 3, pp. 285-292, 2014. 


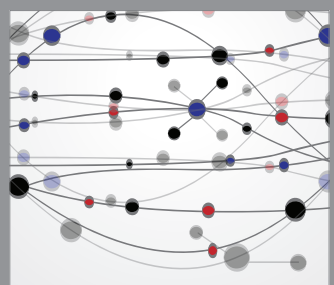

The Scientific World Journal
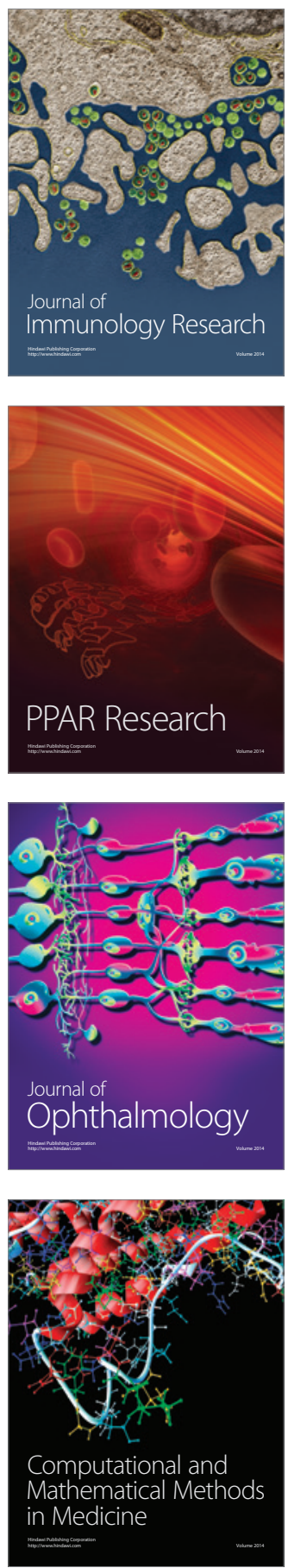

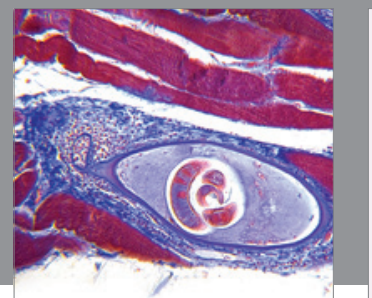

Gastroenterology

Research and Practice
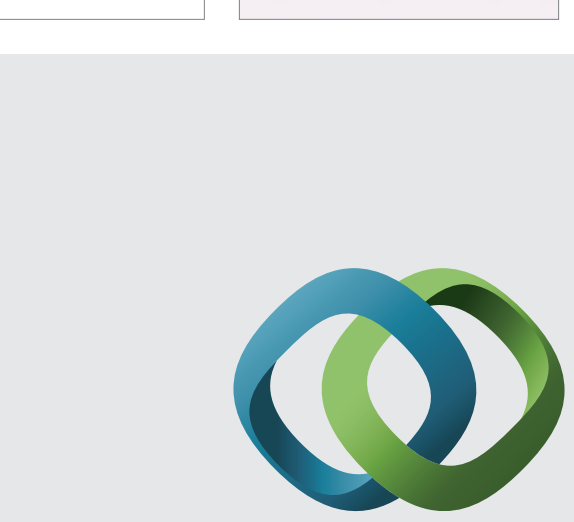

\section{Hindawi}

Submit your manuscripts at

http://www.hindawi.com
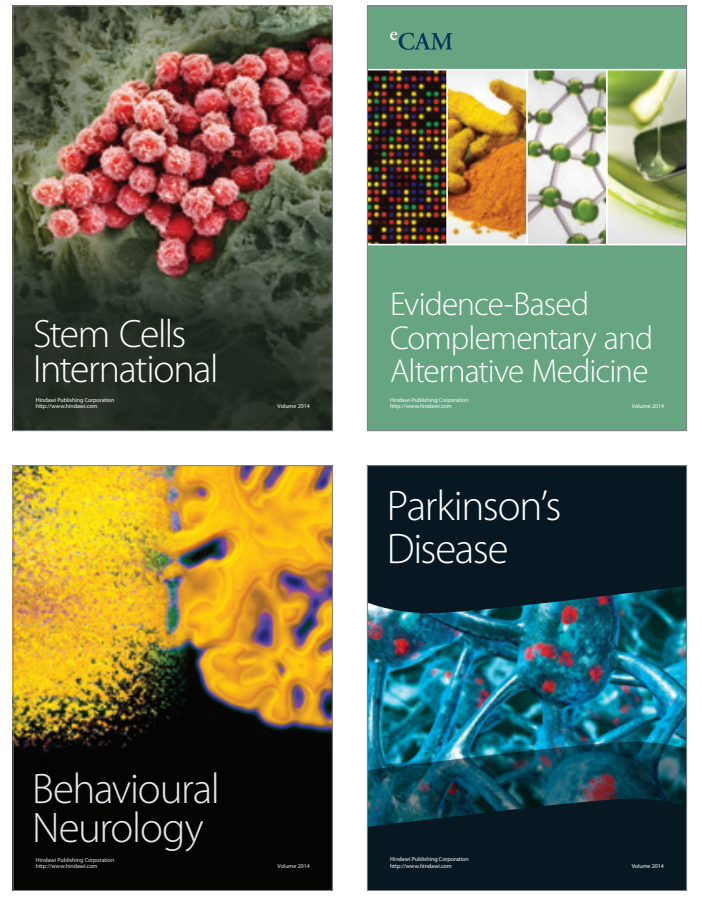
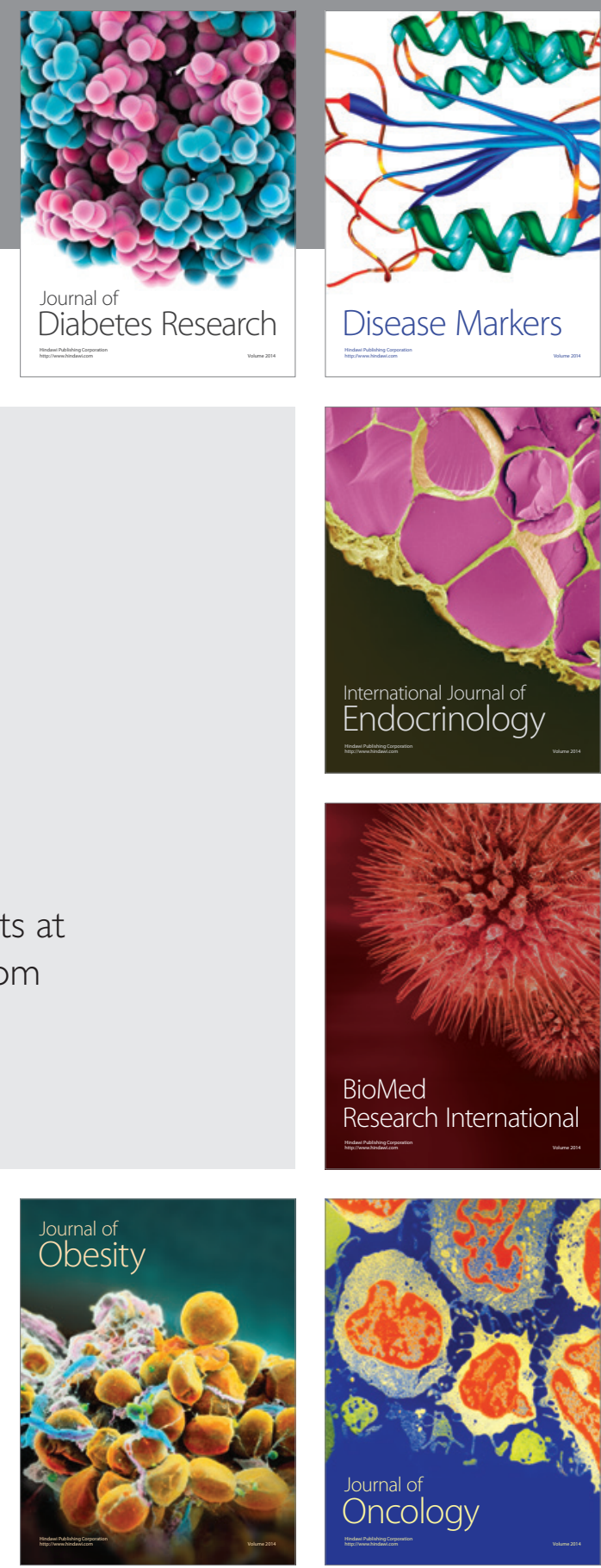

Disease Markers
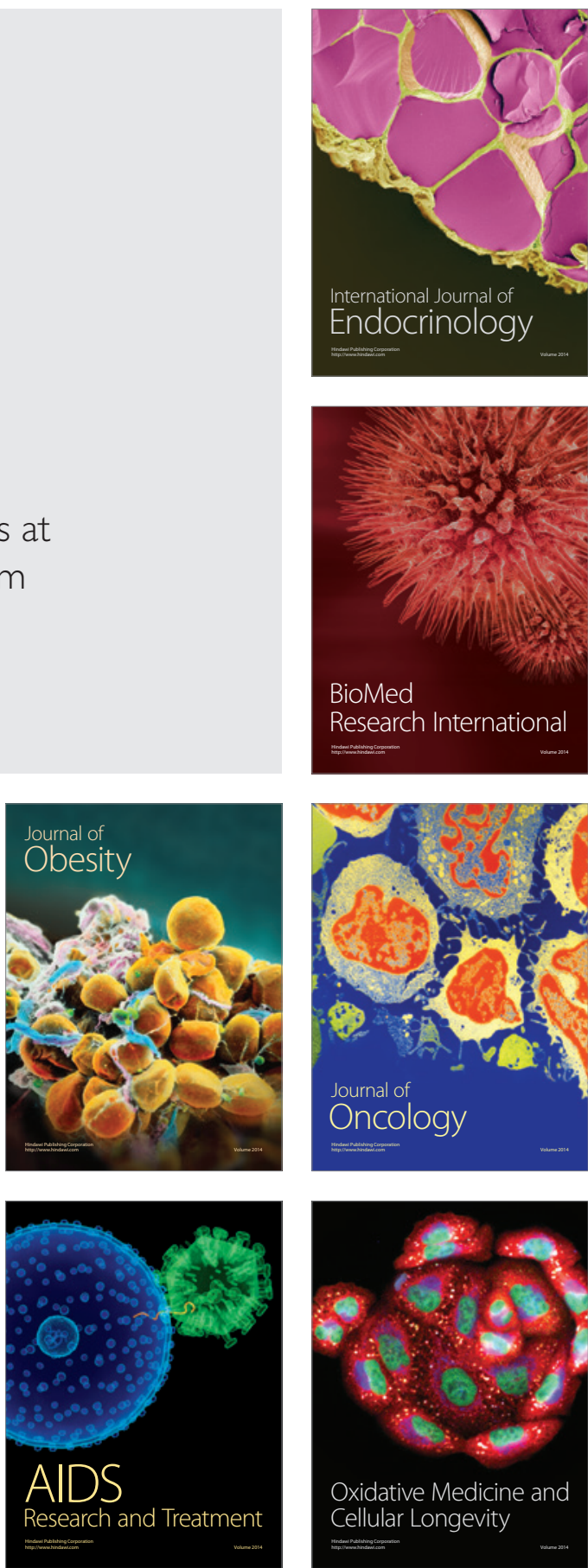